Cahiers
de a 2 Recherche
sur les Droits

Cahiers de la recherche sur les droits fondamentaux

Fondamentaux

Urbanisme et droits fondamentaux

Chronique de jurisprudence de la Cour européenne des droits de l'homme relative aux prisons 2014-2015

A Chronicle of the Case Law of the European Court of Human Rights Concerning Prisons 2014-2015

Jean-Manuel Larralde

(2) OpenEdition

Journals

Édition électronique

URL : https://journals.openedition.org/crdf/603

DOI : $10.4000 /$ crdf.603

ISSN : 2264-1246

Éditeur

Presses universitaires de Caen

Édition imprimée

Date de publication : 1 novembre 2016

Pagination : 147-155

ISBN : 978-2-84133-838-2

ISSN : $1634-8842$

Référence électronique

Jean-Manuel Larralde, «Chronique de jurisprudence de la Cour européenne des droits de l'homme relative aux prisons 2014-2015 », Cahiers de la recherche sur les droits fondamentaux [En ligne], 14 | 2016, mis en ligne le 01 octobre 2019, consulté le 15 novembre 2022. URL : http://

journals.openedition.org/crdf/603; DOI : https://doi.org/10.4000/crdf.603 


\title{
Chronique de jurisprudence de la Cour européenne des droits de l'homme relative aux prisons 2014-2015
}

\author{
Jean-Manuel LARRALDE \\ Professeur de droit public à l'université de Caen Normandie \\ Centre de recherche sur les droits fondamentaux et les évolutions du droit (CRDFED, EA 2132)
}

\author{
I. La légitimité des détentions \\ II. La dignité des détentions \\ III. La prise en charge de la santé des détenus \\ IV. Les moyens du traitement pénitentiaire \\ V. Des voies de recours effectives
}

Si la Convention européenne des droits de l'homme du 4 novembre 1950 ne possède pas de clause comparable à celle de l'article 10 du Pacte onusien relatif aux droits civils et politiques du 16 décembre 1966 qui prévoit que «Toute personne privée de sa liberté est traitée avec humanité et avec le respect de la dignité inhérente à la personne humaine ", les organes strasbourgeois se sont très rapidement saisis de la question du respect des droits de l'homme dans les situations de privation de liberté, tout spécialement dans les prisons. Dès 1962, la Commission européenne des droits de l'homme précise, en effet, que:

[...] même si un requérant se trouve détenu en exécution d'une condamnation qui lui a été infligée en raison de crimes perpétrés au mépris des droits les plus élémentaires de la personne humaine, cette circonstance ne le prive cependant point de la garantie des droits et libertés définis dans la Convention européenne de sauvegarde des droits de l'homme $[\ldots]^{1}$.

La privation de liberté n'est donc pas incompatible avec le statut de requérant devant la Commission (jusqu'en 1998) et la Cour européenne des droits de l'homme (Cour EDH). Bien plus, cette dernière a rappelé en 1984 dans une formule emblématique aujourd'hui bien connue que «la justice ne saurait s'arrêter à la porte des prisons $»^{2}$. Poursuivant l'évolution, l'arrêt Kudla c. Pologne du 26 octobre 2000 précise qu'il incombe aux États de «s'assurer que tout prisonnier est détenu dans des conditions qui sont compatibles avec le respect de la dignité humaine», ce qui implique que "eu égard aux exigences pratiques de l'emprisonnement, la santé et le bien-être du prisonnier [doivent être] assurés de manière adéquate ${ }^{3}$.

À partir de ces lignes directrices, la Cour EDH a progressivement affiné et précisé sa jurisprudence, afin d'imposer des standards protecteurs des personnes incarcérées. La Cour de Strasbourg se situe ici dans l'exacte perspective des Règles pénitentiaires européennes $(\mathrm{RPE})^{4}$ qui rappellent dans leur principe $\mathrm{n}^{\circ} 1$ que «les personnes privées de liberté doivent être traitées dans le respect des droits de l'homme», le principe $n^{\circ} 2$ ajoutant que toutes les personnes privées de liberté conservent «tous les droits qui ne leur ont pas été retirés selon la loi par la décision

1. Commission EDH, 8 mars 1962, Ilse Koch c. RFA, n 1670/61.

2. Cour EDH, 28 juin 1984, Campbell et Fell c. Royaume-Uni, série A, nº 80, $\$ 69$.

3. Cour EDH, 26 octobre 2000, Kudla c. Pologne, $\mathrm{n}^{\circ} 30210 / 96, \$ 94$.

4. Recommandation $\operatorname{Rec}(2006) 2$ du Comité des ministres aux États membres sur les Règles pénitentiaires européennes, adoptée le 11 janvier 2006. 
les condamnant à une peine d'emprisonnement ou les plaçant en détention provisoire».

Sans apporter de décisions spectaculaires ou totalement novatrices, les années 2014-2015 ont néanmoins permis de renforcer la jurisprudence strasbourgeoise sur un certain nombre de points. Celle toujours volumineuse relative aux prisons nous rappelle, en effet, que toute détention doit être légitime (I) et digne (II). Afin de réaliser cette deuxième exigence, il est nécessaire que les autorités pénitentiaires prennent en charge de manière adéquate la santé des détenus (III) et adoptent des techniques de «traitement pénitentiaire» conformes aux exigences des droits de l'homme (IV). Pour qu'elles ne demeurent pas au stade de vœux pieux, ces différentes exigences nécessitent enfin des garanties, qui passent par le respect et le renforcement des voies de recours ouvertes aux personnes détenues $(\mathrm{V})$.

\section{La légitimité des détentions}

Sans renier la place importante de la prison dans l'arsenal répressif, la Cour de Strasbourg a développé une jurisprudence qui cherche à encadrer le recours à la prison, qui ne peut être LA solution unique en matière de sanction pénale. Tout séjour en prison doit être aussi bref que possible, motivé par de strictes exigences de répression de faits graves, et entouré de garanties au profit du condamné.

La durée des détentions (qu'il s'agisse de détentions avant jugement ou de peines privatives de liberté) ne doit pas être excessive comme le rappelle l'arrêt Gallardo Sanchez c. Italie du 24 mars 2015 ( $\left.\mathrm{n}^{\circ} 11620 / 07\right)$ : en maintenant sous écrou extraditionnel le requérant pendant un an et six mois alors que l'affaire ne présentait guère de difficultés, la détention n'était pas justifiée car les autorités étatiques n’ont pas mené la procédure avec la diligence requise. La Cour nous rappelle ici qu'une privation de liberté peut être régulière selon la législation interne tout en étant arbitraire et donc contraire à la Convention. Cette idée d'une «durée légitime» des peines de prison explique que la Cour rejette la conventionnalité des peines incompressibles, qui ne donnent aucun espoir de retour dans la vie civile au condamné. L'arrêt Öcalan c. Turquie ( $\left.n^{\circ} 2\right)$ du 18 mars 2014 rappelle ainsi que, dans les situations de peines de perpétuité, il incombe aux autorités pénales de vérifier que le maintien en détention se justifie toujours

[...] soit parce que les impératifs de répression et de dissuasion ne seront pas encore entièrement satisfaits, soit parce que le maintien en détention de l'intéressé sera justifié par des raisons de dangerosités.

Dans son arrêt Hutchinson c. Royaume-Uni du 3 février 2015 ( $n^{\circ}$ 57592/o8), la Cour reconnaît ainsi l'importante évolution du droit britannique, qui, sous la pression européenne, fait désormais obligation au ministre de la Justice de libérer tout détenu condamné à la perpétuité réelle dont il peut être établi que des «motifs exceptionnels » justifient la libération (l'exercice de ce pouvoir étant soumis au contrôle des juridictions nationales). De telles modifications sont nécessaires dans d'autres États, ce qui a amené la Cour, dans l'affaire László Magyar c. Hongrie du 20 mai 2014 ( $\left.n^{\circ} 73593 / 10\right)$, à demander expressément à la Hongrie de réformer son système de réexamen des peines de perpétuité réelle afin de garantir qu'il soit examiné dans chaque cas si le maintien en détention se justifie par des motifs légitimes et de permettre aux détenus condamnés à la perpétuité réelle de prévoir ce qu'ils doivent faire pour pouvoir bénéficier d'un élargissement, et sous quelles conditions. Ce refus des peines de perpétuité réelle explique également que la Cour juge que l'extradition d'une personne vers un État où elle encourt une peine perpétuelle incompressible est contraire à la Convention ${ }^{6}$. Sans vouloir imposer une durée maximale pour les peines privatives de liberté, la Cour souligne cependant

[...] qu'il se dégage des éléments de droit comparé et de droit international une nette tendance en faveur de l'instauration d'un mécanisme spécial garantissant un premier réexamen dans un délai de vingt-cinq ans au plus après l'imposition de la peine perpétuelle puis des réexamens périodiques?

Les privations de liberté carcérales doivent par ailleurs être limitées aux situations strictement nécessaires et parfaitement motivées. Ainsi une détention provisoire décidée sans aucune motivation par un tribunal et sans aucun délai viole la Convention ${ }^{8}$. De même, une détention provisoire de près de deux ans, prolongée à plusieurs reprises sans que les juges ne tiennent compte des arguments avancés par le requérant en faveur de sa remise en liberté (consistant notamment à dire qu'il avait un casier judiciaire vierge et qu'il n'y avait pas de preuve qu'il constitue un danger pour le public s'il était remis en liberté), violent l'article $5, \$_{3}$, de la Convention'. Une même démarche est retenue par la Cour dans l'arrêt Zayidov c. Azerbaïdjan du 20 février 2014 ( $n^{\circ} 11948 / 08$ ). La Cour de Strasbourg déplore que dans cette affaire de violences conjugales les juges internes ayant examiné les demandes de remise en liberté de l'intéressé n'aient fait aucun cas des arguments tirés de ce qu'il n'avait pas d'antécédents judiciaires, de ce qu'il n'avait jamais tenté de s'enfuir et de ce qu'il avait toujours coopéré avec les enquêteurs. Dans son arrêt Ruiz Rivera c. Suisse du 18 février 2014 ( $\mathrm{n}^{\circ} 8300 / 06$ ), qui concernait une personne placée en internement psychiatrique pour avoir tué et décapité sa femme, la Cour EDH rappelle également qu'aucune privation de liberté d'une personne considérée comme aliénée ne peut être jugée conforme à

5. Cour EDH, 18 mars 2014, Öcalan c. Turquie ( $\left.n^{\circ} 2\right), \mathrm{n}^{\circ}$ 24069/03, 197/04, 6201/06 et 10464/07, \$ 207.

6. Cour EDH, 4 septembre 2014, Trabelsi c. Belgique, $\mathrm{n}^{\circ}$ 140/10.

7. Cour EDH, 13 novembre 2014, Bodein c. France, $\mathrm{n}^{\circ}$ 40014/10, $\$ 55$.

8. Cour EDH, 7 mai 2015, Aleksandr Dmitriyev c. Russie, $\mathrm{n}^{\circ}$ 12993/05.

9. Cour EDH, 11 février 2014, Karoly c. Roumanie, $\mathrm{n}^{\circ}$ 33682/05. 
la Convention lorsqu'elle est décidée sans consulter l'avis suffisamment récent d'un médecin expert. Or en l'espèce, l'expertise psychiatrique datait de plus de trois ans. La Cour considère que les autorités nationales auraient dû avoir recours à un avis médical tiers plus récent pour décider ou non du maintien en détention de l'intéressé ${ }^{10}$.

Plusieurs affaires ont également permis à la Cour de réaffirmer que la détention ne peut être un outil des États pour museler le débat démocratique. Dans les affaires Nedim Şener c. Turquie et Şik c. Turquie du 8 juillet 2014, elle conclut en effet que la privation de liberté infligée à deux journalistes d'investigation était

[...] susceptible de créer un climat d'autocensure pour tous les journalistes d'investigation envisageant d'effectuer des recherches et de faire des commentaires sur les comportements et agissements des organes étatiques ${ }^{11}$.

Le placement et le maintien des requérants en détention provisoire pendant plus d'un an ne répondaient donc pas à un besoin social impérieux et n'étaient pas de ce fait nécessaires dans une société démocratique ${ }^{12}$. Ce refus d'une prison "arme contre la liberté d'expression » est également au cœur de l'arrêt Taranenko c. Russie du 15 mai 2014, qui concernait le cas d'une étudiante en sociologie qui avait participé dans le cadre de ses recherches à une manifestation contre Vladimir Poutine, ce qui avait entraîné sa mise en détention provisoire pendant près d'un an et une condamnation à trois ans de prison avec sursis, pour participation à des troubles de masse. Voyant dans la manifestation de décembre 2004 l'expression de revendications politiques légitimes dans une démocratie, la Cour européenne relève la « durée de la détention préventive et l'exceptionnelle sévérité de la sanction en cause ${ }^{13}$. Sans contester que les faits puissent justifier une sanction pénale (une arrestation pouvait en l'espèce être justifiée en raison des troubles à l'ordre public survenus), elle conclut que la peine de prison avec sursis qui a été infligée n'était pas proportionnée au but légitime poursuivi et a présenté un effet dissuasif, non seulement pour la requérante, mais aussi pour toute personne souhaitant participer à une manifestation. Dans le même sens, des peines de prison de deux à quatre ans ferme constituent des sanctions disproportionnées, même à l'encontre de manifestants ayant participé à des affrontements violents avec la police et incité à l'usage de la force les participants à une manifestation politique publique ${ }^{14}$. La Cour a également jugé qu'une peine de treize ans d'emprisonnement pour avoir déversé de la peinture sur des statues d'Atatürk (et donc avoir «porté atteinte» à sa mémoire) était manifestement disproportionnée ${ }^{15}$; et même que dix jours de détention pour un acte de protestation politique dirigé contre le président ukrainien Yanukovych est une peine excessive ${ }^{16}$.

Ces différents arrêts ne signifient toutefois pas que les juges de Strasbourg se soient arrogé le droit de contrôler librement le «tarif» des sanctions pénales infligées, comme le montre l'arrêt Gough c. Royaume-Uni du 28 octobre 2014 ( $\left.n^{\circ} 49327 / 11\right)$. Dans cet arrêt, la Cour n'a pas retenu de violation de la Convention dans la situation du « randonneur nu» écossais, condamné à de multiples peines de prison (atteignant au total une durée cumulée de plus de sept ans). Si la Cour juge qu'apparaître nu en public était certes pour l'intéressé une manière d'exprimer son opinion sur le caractère inoffensif du corps humain et que l'impact cumulé des nombreuses peines d'emprisonnement est sévère, elle insiste néanmoins sur la propre responsabilité de l'intéressé quant aux condamnations et peines prononcées face à son refus délibéré de respecter la loi durant un certain nombre d'années et rappelle la large marge d'appréciation dont disposent les États pour protéger les bonnes mœurs...

Enfin, concernant les garanties indispensables, les affaires Nedim Şener c. Turquie et Şikc. Turquie (précitées) constituent un bon exemple des exigences strasbourgeoises. Dans ces arrêts, la Cour EDH juge que le maintien de deux journalistes d'investigation en détention provisoire pendant plus de deux ans viole la Convention. La Cour considère ici qu'en reprochant aux requérants dès le début de l'enquête des faits de «crimes graves de terrorisme» (ils étaient accusés d'avoir apporté un soutien à l'organisation criminelle Ergenekon qui avait fomenté un coup d'État en 2013) et en présumant la nécessité de leur maintien en détention provisoire, les autorités ont motivé leur décision d'une manière qui n'est ni «pertinente» ni «suffisante» pour justifier une telle durée. En outre, leurs avocats n'ont pu correctement contester la légalité de la détention (la Cour insistant depuis quelques années sur l'existence de voies de recours effectives au profit des condamnés ${ }^{17}$ ).

\section{La dignité des détentions}

Sans beaucoup de surprise, les années 2014-2015 ont encore amené un ensemble de jurisprudences concernant l'indignité des conditions de détention ${ }^{18}$. Ainsi, dans les deux arrêts du 20 février 2014, Firstov c. Russie (no 42119/o4) et

10. Dans le même sens, voir Cour EDH, 18 juin 2015, Yaikov c. Russie, $\mathrm{n}^{\circ}$ 39317/o5.

11. Cour EDH, 8 juillet 2014, Nedim Şener c. Turquie et Şik c. Turquie, $\mathrm{n}^{\circ} 38270 / 11$ et 53413/11, $\$ 122$ et 111.

12. Dans un sens comparable, voir Cour EDH, 18 juin 2015, Mehdiyev c. Azerbaïdjan, $\mathrm{n}^{\circ}$ 59075/o9, à propos d'un journaliste en détention administrative pour une durée de quinze jours dans des conditions contraires à l'article 3 de la Convention.

13. Cour EDH, 15 mai 2014, Taranenko c. Russie, $\mathrm{n}^{\circ}$ 19554/05, $\$ 96$ (notre traduction).

14. Cour EDH, 6 octobre 2015, Karpyuk et autres c. Ukraine, $\mathrm{n}^{\circ} 30582 / 04$ et 32152/04.

15. Cour EDH, 21 octobre 2014, Murat Vural c. Turquie, $\mathrm{n}^{\circ} 9540 / 07$.

16. Cour EDH, 30 octobre 2014, Shvydka c. Ukraine, $\mathrm{n}^{\circ}$ 17888/12.

17. Voir infra.

18. Voir également, inter alia, Cour EDH, 8 juillet 2014, Harakchiev et Tolumov c. Bulgarie, $\mathrm{n}^{\circ}$ 15018/11 et 61199/12; 25 juin 2015, Anatoliy Kuzmin c. Russie, $\mathrm{n}^{\circ}$ 28917/05; 17 novembre 2015, Dimitrov et Ribov c. Bulgarie, $\mathrm{n}^{\circ} 34846 / 08 ; 17$ novembre 2015, Radev c. Bulgarie, $\mathrm{n}^{\circ} 37994 / 09$. 
Shishkov c. Russie (n ${ }^{\circ}$ 26746/05), la Cour retient l'humidité des cellules, l'absence de literie et de place pour dormir (chacun devant s'installer à tour de rôle sur le sol), l'absence de nourriture et d'hygiène adéquates, ainsi que d'installations sanitaires (les codétenus devaient utiliser un seau en guise de toilettes), pour retenir la violation de l'article 3 de la Convention ${ }^{19}$. Au-delà de ces malheureusement classiques exemples du délabrement général des conditions de détention, la Cour a également condamné des situations plus spécifiques, comme le fait d'exposer un détenu à des produits chimiques dangereux lors de la désinfection de sa cellule ${ }^{20}$, ou encore le transport dans des conditions inhumaines par fourgon cellulaire et par train lors de transferts entre deux pénitenciers ${ }^{21}$. Le même constat de violation de l'article 3 de la Convention a été effectué pour un détenu transporté entre la maison d'arrêt et le tribunal à plus de cent reprises dans un véhicule de marchandises sans fenêtres ni ceintures de sécurité, pour être ensuite détenu dans les petites cellules surpeuplées d'un tribunal où il passa à chaque fois jusqu'à huit heures ${ }^{22}$.

Participant à la lutte pour des conditions de détention dignes, la Cour a également eu l'occasion de dénoncer à nouveau les situations de surpeuplement carcéral ${ }^{23}$, comme le montre l'arrêt Muršićc c. Croatie du 12 mars 2015 $\left(n^{\circ} 7334 / 13\right)$, qui constitue une bonne synthèse des positions actuelles de la Cour en la matière. Certes le requérant n'a pas toujours bénéficié des $3 \mathrm{~m}^{2}$ d'espace personnel, qui semblaient constituer la «norme minimale» depuis l'arrêt Ananyev et autres c. Russie du 10 janvier 2012 ( ${ }^{\circ}$ 42525/07 et 60800/08), en deçà de laquelle la Cour concluait automatiquement à la violation de la Convention. Mais l'arrêt du 12 mars 2015 relève que le requérant était autorisé à circuler librement trois heures par jour hors de sa cellule qui était pourvue d'une ouverture laissant passer sans entrave la lumière naturelle et l'air extérieur, ainsi que d'un point d'eau potable, d'un lit individuel et que rien ne l'empêchait de circuler librement à l'intérieur de la cellule. En outre, plusieurs activités étaient accessibles à l'extérieur des cellules telles que des activités sportives et la bibliothèque. Il n'y avait donc pas, en l'espèce, violation de l'article 3 de la Convention. Les juges de Strasbourg calquent ici leur position sur celle du Comité européen pour la prévention de la torture (CPT) qui a précisé depuis plus de vingt ans que la situation s'avère préoccupante lorsque l'on constate
[...] dans un même établissement une combinaison de surpeuplement, de régimes pauvres en activités et d'un accès inadéquat aux toilettes ou locaux sanitaires. L'effet cumulé de telles conditions peut s'avérer extrêmement néfaste pour les prisonniers ${ }^{24}$.

L'existence de nombreuses conditions de détention indignes a généré un contentieux répétitif, qui a amené la Cour à utiliser sa technique de l'arrêt pilote pour contraindre les États concernés à entreprendre des réformes structurelles. Dans ses arrêts du 16 septembre 2014, Stella c. Italie et dix autres requêtes ( $\mathrm{n}^{\circ}$ 49169/o9) et Rexhepi c. Italie et sept autres requêtes ( $\mathrm{n}^{\circ} 4718 \mathrm{o} / 10$ ), la Cour a ainsi pu vérifier que l'État italien, conformément aux préconisations exprimées dans l'arrêt pilote Torreggiani et autres $c$. Italie du 8 janvier 2013 ( $n^{\circ}$ 43517/09, 46882/o9, 55400/09, 57875/o9, $61535 / 09,35315 / 10$ et $37818 / 10$ ), a opéré des modifications du droit interne, en prévoyant tout à la fois la construction de nouveaux bâtiments et une meilleure répartition des détenus, l'accroissement des réductions de peine pour bonne conduite, l'augmentation des mesures alternatives à la détention, l'institution d'un médiateur national des personnes détenues ${ }^{25}$, la refonte des sanctions applicables aux délits mineurs (notamment en ce qui concerne la répression des infractions à la législation sur les stupéfiants), l'accroissement de la liberté de mouvement des détenus en dehors de leurs cellules, un accès plus facile au travail et une augmentation des visites familiales, la mise en place d'un système informatique de gestion en temps réel des places en établissement, ainsi qu'une nouvelle voie de recours permettant désormais à tout détenu de présenter devant le juge de l'application des peines une réclamation portant sur le non-respect par l'Administration des dispositions de la loi pénitentiaire entraînant une atteinte grave à l'exercice de ses droits, dont le droit à disposer d'un espace vital suffisant et à bénéficier de conditions matérielles de vie convenables. Cette technique de l'arrêt pilote a été reprise dans l'arrêt Vasilescu c. Belgique du 25 novembre 2014 (no 64682/12). Constatant que les problèmes découlant de la surpopulation carcérale en Belgique ainsi que les problèmes d'hygiène et de vétusté des établissements pénitentiaires revêtent un caractère structurel, la Cour recommande à la Belgique d'envisager l'adoption de mesures générales afin, d'une part, de garantir aux détenus des conditions de détention conformes à l'article 3 de la Convention et, d'autre part, de leur offrir un recours visant à empêcher la continuation

19. Sur les conditions sanitaires insatisfaisantes, voir également les arrêts Cour EDH, 10 mars 2015, Halil Adem Hasan c. Bulgarie, $\mathrm{n}^{\circ}$ 4374/o5; 21 mai 2015, Yengo c. France, nº 50494/12.

20. Cour EDH, 17 juin 2014, Zamfirachi c. Roumanie, $\mathrm{n}^{\circ}$ 70719/10.

21. Cour EDH, 13 mars 2014, Andrey Yakovenko c. Ukraine, $\mathrm{n}^{\circ}$ 63727/11. Le requérant, souffrant de problèmes vasculaires et cardiaques, a dû longuement voyager dans des compartiments surchargés, dans des positions inconfortables, dans des wagons sans climatisation en été, rendant la respiration difficile, avec un accès aux toilettes seulement toutes les quatre heures.

22. Cour EDH, 11 juin 2015, Tychko c. Russie, n 56097/o7. Voir également Cour EDH, 22 septembre 2015, Ilkin c. Russie, $\mathrm{n}^{\circ}$ 12436/11.

23. Sur les affaires relatives au surpeuplement carcéral, voir, inter alia, Cour EDH, 6 mars 2014, Gorbulya c. Russie, $\mathrm{n}^{\circ} 31535 / 09 ; 18$ mars 2014 , Stark c. Roumanie, $\mathrm{n}^{\circ}$ 31968/07; 16 juin 2015, Constantin Nistor c. Roumanie, n 35091/12; 16 juin 2015, Ghiroga c. Roumanie, $\mathrm{n}^{\circ} 53168 / 12 ; 23$ juin 2015 , Costel Gaciu c. Roumanie, $\mathrm{n}^{\circ}$ 39633/10; 25 juin 2016, Lutanyuk c. Grèce, $\mathrm{n}^{\circ}$ 60362/13; 6 octobre 2015, Sergeyev c. Russie, $\mathrm{n}^{\circ} 41090 / 05 ; 27$ octobre 2015 , Brânduşe c. Roumanie (n² 2), nº 39951/o8; 24 novembre 2015, Verdeș c. Roumanie, n 6215/14.

24. CPT, «Normes du CPT», extrait du Deuxième rapport général, CPT/Inf (92) 3, \$50.

25. Comparable au contrôleur général des lieux de privation de liberté mis en place en France par la loi no $2007-1545$ du 30 octobre 2007 instituant un contrôleur général des lieux de privation de liberté. 
d'une violation alléguée ou à leur permettre d'obtenir une amélioration de leurs conditions de détention. La même démarche a été suivie à l'encontre de la Hongrie avec l'arrêt Varga et autres c. Hongrie du 10 mars 2015 ( $n^{\circ}$ 14097/12).

\section{La prise en charge de la santé des détenus}

Les arrêts Sandu Voicu c. Roumanie du 3 mars 2015 $\left(n^{\circ} 45720 / 11\right)$ relatif à un détenu épileptique et atteint de multiples affections fonctionnelles et de déficience de la colonne vertébrale, Davtyan c. Arménie du 31 mars 2015 ( $\mathrm{n}^{\circ}$ 29736/06) relatif à un détenu souffrant d'une tumeur aux cordes vocales, ou encore Veretco c. République de Moldova du 7 avril 2015 ( $n^{\circ}$ 679/13) relatif à un détenu souffrant de fractures des côtes et d'une pneumonie, confirment que la Cour exige que les autorités pénitentiaires prodiguent avec diligence les soins adéquats aux détenus malades. Ainsi, se contenter de faire examiner un détenu souffrant d'une grave maladie cérébrale qui entraîne des symptômes incluant des maux de tête sévères, des crises d'épilepsie, des nausées et des insomnies par un auxiliaire médical et un psychiatre de la prison, alors que ce prisonnier avait besoin d'une intervention neurochirurgicale, viole l'article 3 de la Convention ${ }^{26}$.

Cette exigence de soins concerne tout spécialement les détenus que la Cour de Strasbourg qualifie de «vulnérables». Ainsi en est-il des toxicomanes, pour lesquels les États ont l'obligation d'assurer une protection générale. Toutefois, les autorités ne peuvent être tenues pour responsables de la mort par overdose d'un détenu, si elles ont satisfait à leur obligation d'agir contre le trafic de drogue en milieu carcéral par des mesures suffisantes ${ }^{27}$. Les personnes souffrant de troubles psychiatriques constituent une autre catégorie de détenus vulnérables. À leur égard, des protocoles de soins spécifiques doivent être mis en place, si possible dans des établissements hospitaliers spécialisés ${ }^{28}$. Cette exigence n'est évidemment pas respectée pour un détenu souffrant du syndrome de Ganser (appelé aussi «psychose de prison») ayant subi des transferts répétés (quarante-trois en six ans!), accompagnés de longues périodes d'isolement et de mesures de sécurité renforcées ${ }^{29}$. Constituent également des personnes vulnérables, les détenus séropositifs (ne présentant pas de symptômes de la maladie), qui ne doivent pas subir un isolement, hormis pour leur assurer des conditions de détention plus appropriées avec leurs besoins spécifiques. En conséquence,

Si un détenu séropositif [doit] être séparé des autres détenus, il devrait être placé dans un endroit en adéquation avec ses besoins médicaux et son bien-être ${ }^{30}$.

Et non dans un hôpital pénitentiaire à proximité de détenus souffrant de maladies telles que la tuberculose ou l'hépatite... Les personnes handicapées sont aussi des détenus vulnérables aux yeux de la Cour. Les détenir dans des lieux non adéquats, où elles ne peuvent accéder aux toilettes ou à la douche qu'en demandant l'aide d'autres détenus, et où la configuration des bâtiments ne permet aucun exercice en plein air (le fauteuil roulant avait été placé loin de l'intéressé dans le dortoir pour des raisons de sécurité) viole l'article 3 de la Convention ${ }^{31}$. L'arrêt Helhal c. France du 19 février 2015 réaffirme d'ailleurs la volonté des juges strasbourgeois d'imposer aux États une accessibilité architecturale des bâtiments pour tous les types de détenus qui s'y trouvent incarcérés. La Cour relève ici que le requérant, victime d'une fracture de la colonne vertébrale entraînant une paraplégie des membres inférieurs et une incontinence urinaire et anale, et contraint de se déplacer principalement en chaise roulante, se trouve dans une situation de « dépendance [qui] l'expose à des situations humiliantes vis-à-vis de [son] auxiliaire et des autres détenus du fait de son incontinence $»^{32}$ et pour effectuer sa toilette. On peut par ailleurs souligner que la Cour juge ici inappropriée la disposition introduite par la loi pénitentiaire $\mathrm{n}^{\circ}$ 2009-1436 du 24 novembre 2009 , permettant à toute personne détenue dans une situation de handicap de désigner un aidant de son choix $^{33}$. Selon elle, on ne peut

[...] approuver une situation dans laquelle le personnel d'une prison se dérobe à son obligation de sécurité et de soins vis-à-vis des détenus les plus vulnérables en faisant peser sur leurs compagnons de cellule la responsabilité de leur fournir une assistance quotidienne ou, le cas échéant, des soins d'urgence; cette situation engendre de l'angoisse et les place dans une position d'infériorité vis-à-vis des autres détenus ${ }^{34}$.

Ce refus est conforme à l'attitude générale de la Cour qui, à plusieurs reprises, a exprimé son opposition quant à la pratique de certains établissements pénitentiaires consistant à confier à des personnes non qualifiées la responsabilité d'assister un individu malade ou handicapé ${ }^{35}$.

26. Cour EDH, 9 janvier 2014, Budanov c. Russie, n 66583/11.

27. Cour EDH, 8 avril 2014, Marro et autres c. Italie, $\mathrm{n}^{\circ}$ 29100/07. Voir également Cour EDH, 8 octobre 2015, Sellal c. France, $\mathrm{n}^{\circ} 32432 / 13$, qui conclut à un constat de non-violation de l'article 2 de la Convention après le suicide en détention d'un détenu atteint de schizophrénie, mais pour lequel la maladie ne s'accompagnait pas de tendances suicidaires et pour lequel aucun antécédent ou élément de conduite récente ne pouvait laisser prévoir l'autolyse.

28. Cour EDH, 25 février 2014, Gheorghe Predescu c. Roumanie, no 19696/10.

29. Cour EDH, 17 novembre 2015, Bamouhammad c. Belgique, $\mathrm{n}^{\circ}$ 47687/13.

30. Cour EDH, 9 juillet 2015, Martzaklis et autres $c$. Grèce, n ${ }^{\circ} 20378 / 13, \S 71$

31. Cour EDH, 6 février 2014, Semikhvostov c. Russie, $\mathrm{n}^{\circ} 2689 / 12$.

32. Cour EDH, 19 février 2015, Helhal c. France, ${ }^{\circ} 10401 / 12, \$ 62$.

33. Art. R. 57-8-6 du Code de procédure pénale, issu du décret nº 2010-1634 du 23 décembre 2010.

34. Cour EDH, 19 février 2015, Helhal c. France, $\$ 62$.

35. Voir également Cour EDH, 6 février 2014, Semikhvostov c. Russie, nº 2689/12. 
Ce même arrêt Helhal confirme par contre qu'il n'existe selon la Cour aucune obligation pour les États de libérer un détenu pour des raisons médicales. Le caractère adéquat des soins est le critère essentiel, qu'ils soient prodigués à l'intérieur de la prison, ou dans un établissement extérieur ${ }^{36}$. Toutefois, lorsque des rapports ou certificats médicaux concluent de manière concordante à l'incompatibilité de l'état de santé d'un détenu avec le régime de détention auquel il est soumis, les autorités pénitentiaires ont l'obligation de l'autoriser à suivre des soins à l'extérieur de l'établissement ${ }^{37}$.

L'arrêt McDonnell c. Royaume-Uni du 9 décembre 2014 $\left(n^{\circ} 19563 / 11\right)$ a enfin rappelé que tout décès en détention (notamment en raison d'un défaut de soins) nécessite que les autorités étatiques mettent en place une enquête rapide, impartiale et efficace. Une certaine lenteur entraîne ainsi une condamnation automatique de l'État pour violation de l'article 2 de la Convention (droit à la vie).

\section{Les moyens du traitement pénitentiaire}

Euvrant pour une prison plus humaine, la Cour de Strasbourg rappelle constamment que la mission de la prison n'est plus aujourd'hui le «dressement» des siècles passés, mais le «traitement pénitentiaire», qui nécessite de limiter, voire d'interdire certaines pratiques ou mesures en détention.

L'arrêt Tali c. Estonie du 13 février 2014 (nº6393/10) a ainsi permis à la Cour de rappeler sa position concernant l'usage de la force en détention ${ }^{38}$. Refusant d'obéir aux gardiens qui lui demandaient de leur remettre son matelas, le requérant, qui purgeait une peine de prison à perpétuité pour meurtre, a été envoyé de manière particulièrement violente en cellule disciplinaire. L'enquête diligentée par l'administration pénitentiaire démontre que les gardiens l'ont menotté, frappé avec une matraque télescopique, lui occasionnant de fortes douleurs aux côtes, lui ont aspergé le visage de gaz poivre (sans aucune sommation) et l'ont sanglé sur un lit de contention pendant trois heures et demie. Pour les juridictions internes, ces incidents n'ont démontré aucun usage disproportionné de la force, à l'égard d'un détenu insoumis et particulièrement violent. La Cour EDH, s'appuyant sur les travaux du CPT, rappelle avec fermeté que l'utilisation d'un spray au gaz poivre dans un espace confiné entraîne des effets particulièrement néfastes pour la santé, et ne constitue pas un moyen approprié pour immobiliser un détenu, à partir du moment où les gardiens pouvaient en utiliser d'autres moins dangereux. Cette ligne jurisprudentielle défendue par la Cour de Strasbourg explique également qu'elle ne reconnaît pas conforme à la Convention une intervention particulièrement violente des forces de l'ordre (lors de laquelle douze détenus trouvèrent la mort et une cinquantaine furent blessés, certains par arme à feu), pour faire cesser une grève de la faim de protestation contre un projet de prison où les unités résidentielles carcérales seraient plus petites pour les détenus ${ }^{39}$.

Les juges de Strasbourg rappellent par ailleurs que les moyens de contrainte (tels qu'un lit de contention) doivent être strictement justifiés par les circonstances et ne peuvent en aucun cas constituer une punition. Ils doivent seulement éviter des automutilations, ou protéger des codétenus ou assurer la sécurité de l'établissement. Cette question de la contention était au cœur de l'affaire Dimcho Dimov c. Bulgarie du 16 décembre 2014 ( ${ }^{\circ}$ 57123/o8), dans laquelle la Cour condamne l'État pour avoir menotté à son lit (par les poignets et chevilles) pendant neuf jours consécutifs (durant lesquels il ne fut détaché que trois fois par jour pour aller aux toilettes et prendre ses repas) un détenu souffrant de troubles comportementaux et qui avait fait part aux autorités pénitentiaires de sa volonté de s'automutiler.

Dans toutes ces situations de violations alléguées de l'article 3 de la Convention, la Cour exige que l'État diligente une enquête rapide, effective et impartiale, sous peine de se voir automatiquement condamné pour violation de la Convention, que les faits aient été prouvés ou non ${ }^{40}$. C'est également sur l'État que repose la charge de la preuve, à qui il incombe notamment de démontrer que l'usage de la force était proportionné et légitime ${ }^{41}$.

Reprenant la position désormais habituelle de la Cour (et des RPE) selon lesquelles la prison doit garantir aux détenus

[...] des conditions de détention qui ne portent pas atteinte à la dignité humaine et offrir des occupations constructives et une prise en charge permettant la préparation à leur réinsertion dans la société $[\ldots]^{42}$

les arrêts rendus en 2014 et 2015 rappellent également que les exigences sécuritaires n'autorisent que des dispositifs nécessaires et proportionnés. Ainsi, les mesures ne visant qu'à humilier les personnes privées de leur liberté ne sont évidemment pas compatibles avec les exigences d'une société démocratique, comme le relève l'arrêt Svinarenko et Slyadnev c. Russie du 17 juillet 2014 ( $\mathrm{n}^{\circ}$ 32541/o8), dans lequel la Grande Chambre rappelle que la pratique consistant à mettre les personnes en détention provisoire dans

36. Cour EDH, 9 septembre 2014, Carrella c. Italie, $\mathrm{n}^{\circ}$ 33955/07; 16 avril 2015, Papastavrou c. Grèce, $\mathrm{n}^{\circ}$ 63054/13.

37. Cour EDH, 11 février 2014, Contrada c. Italie ( $\left.n^{\circ} 2\right), \mathrm{n}^{\circ} 7509 / 08$.

38. L'utilisation de la force est également au cœur de l'arrêt du 11 février 2014, Sapožkovs c. Lettonie, no 8550/o3, concernant un détenu violemment battu lors d'un transfert entre deux établissements; voir également les arrêts du 28 avril 2015, Milić et Nikezić c. Monténégro, n $54999 / 10$ et 10609/11, concernant des violences infligées par des gardiens lors de la fouille d'une cellule.

39. Cour EDH, 26 mai 2015, Songül İnce et autres c. Turquie, $\mathrm{n}^{\circ}$ 25595/08 et 34252/10.

40. Cour EDH, 3 juin 2014, Yiğitdoğan c. Turquie et Habimi et autres c. Serbie, $\mathrm{n}^{\circ}$ 72174/10 et no 19072/o8; 7 juillet 2015, Kardišauskas c. Lituanie, $\mathrm{n}^{\circ} 62304 / 12$.

41. Cour EDH, 6 octobre 2015, Kavakhoğlu et autres c. Turquie, $\mathrm{n}^{\circ}$ 15397/02.

42. Recommandation Rec (2006) 2 du Comité des ministres aux États membres sur les Règles pénitentiaires européennes, préambule. 
des cages de métal au cours des audiences pendant leur procès constitue un traitement dégradant injustifiable, contraire à la dignité humaine, qui viole donc l'article 3 de la Convention. La Cour voit également dans le menottage de deux détenus condamnés à perpétuité, au cours d'une audience dans un milieu sécurisé (à l'intérieur de la prison) et en la présence de gardiens de la prison, un traitement dégradant ${ }^{43}$. De même pour les fouilles, qui doivent être mesurées et justifiées. Cette analyse est au cœur de l'arrêt Ślusarczyk c. Pologne du 28 octobre 2014 (n 23463/o4) qui concernait un requérant classé «détenu dangereux" et soumis à des mesures de haute sécurité, y compris des fouilles corporelles quotidiennes et la pose de chaînes lorsqu'il quittait sa cellule. Pour la Cour, ces contraintes (qui ont duré environ six mois), qui concernaient un détenu déjà soumis à des strictes mesures de surveillance, et qui n'apparaissaient pas motivées par des exigences de sécurité particulières, ont porté atteinte à la dignité de l'intéressé, et lui ont causé des sentiments d'infériorité, d'angoisse et de détresse, allant au-delà des souffrances inhérentes à toute privation de liberté. Si

La Cour est consciente de la nécessité d'assurer la sécurité dans des institutions où les personnes sont privées de leur liberté, elle considère cependant que des mesures fortement invasives et potentiellement humiliantes telles que des fouilles à corps ou des palpations exigent une justification plausible ${ }^{44}$.

Les États doivent également veiller à ne pas porter atteinte de manière excessive au droit à la vie privée et familiale des prisonniers comme le montrent les arrêts Nusret Kaya et autres c. Turquie du 22 avril 2014 ( $\mathrm{n}^{\circ}$ 43750/o6, 43752/o6, 32054/o8, 37753/o8 et 60915/08) dans lesquels la Cour juge que la restriction imposée aux détenus turcs concernant l'usage du kurde dans leurs conversations téléphoniques ne respecte pas les exigences de l'article 8 de la Convention européenne des droits de l'homme. Soulignant que le kurde fait partie des langues couramment parlées en Turquie, la Cour a jugé que les restrictions imposées aux requérants ne pouvaient passer pour nécessaires dans une société démocratique. Même à l'égard des détenus condamnés à perpétuité, la peine privative de liberté ne peut aboutir à un isolement quasi total vis-à-vis des proches: un régime pénitentiaire autorisant seulement deux brèves visites familiales annuelles emporte violation du droit du détenu au respect de sa vie familiale, en empêchant notamment celui-ci d'établir des liens pendant la petite enfance de son enfant ${ }^{45}$.

Ces différentes jurisprudences ne doivent toutefois pas laisser à penser que la Cour de Strasbourg censure systématiquement des conditions de détention particu- lièrement rudes. Amenée à statuer sur le sévère régime d'emprisonnement infligé à Abdullah Öcalan, chef historique du parti (illégal) des travailleurs du Kurdistan, la Cour prend en considération le degré de dangerosité de l'intéressé pour accepter un régime carcéral particulièrement rude et dérogatoire pour de longues durées. Elle insiste toutefois sur l'importance des moyens de communication, qui permettent de «réduire les effets néfastes de l'isolement social», et dont l'accès ne peut être restreint qu'en raison de «justifications convaincantes » ${ }^{46}$. De même, de longs développements sont consacrés à l'importance des possibilités de communication pour de tels détenus, tant avec le personnel de la prison (et tout spécialement le personnel médical) qu'avec les codétenus, les avocats, ainsi que la famille et les proches ${ }^{47}$.

Au-delà de la limitation des dispositifs les plus attentatoires à la dignité des détenus, la Cour cherche également à promouvoir les conditions d'un véritable traitement pénitentiaire. Dans son arrêt Velev c. Bulgarie du 27 mai 2014 ( $\left.\mathrm{n}^{\circ} 16032 / 07\right)$, la Cour souligne ainsi que, même si les détenus ne bénéficient pas d'un droit illimité à l'éducation en détention, tout refus d'accès aux activités éducatives doit être explicitement motivé par des justifications convaincantes. Cette volonté de promotion des activités éducatives en détention ne signifie pas pour autant que la Cour considère les prisonniers comme des citoyens ordinaires, ainsi que le démontrent les décisions $S$. $S$. et autres c. Royaume-Uni et F. A. et autres c. Royaume-Uni du 21 mai 2015 ( $n^{\circ} 40356 / 10$ et 54466/10), dans lesquelles elle reconnaît aux États européens une large marge d'appréciation pour déterminer les personnes (et notamment les condamnés) ayant droit ou non à des prestations sociales. De manière assez étonnante, la Cour valide le raisonnement avancé par le gouvernement selon lequel le versement de prestations équivaudrait à un double avantage, étant donné que l'État répond déjà aux besoins fondamentaux des détenus internés dans des établissements pénitentiaires ou psychiatriques (le gouvernement estimait également que le non-versement de prestations doit être vu comme un aspect de la sanction pénale infligée aux requérants).

\section{Des voies de recours effectives}

Justiciables de plein droit, les détenus le sont d'abord parce qu'ils ne peuvent être condamnés sur la base de faux aveux qui auraient été obtenus au moyen de pressions psychologiques et de mauvais traitements physiques par la police. Dans leur arrêt Yevgeniy Petrenko c. Ukraine du 29 janvier 2015 ( $\left.n^{0} 55749 / 08\right)^{48}$, les juges strasbourgeois

43. Cour EDH, 27 mai 2014, Radkov et Sabev c. Bulgarie, no 18938/o7 et 36069/o9.

44. Cour EDH, 15 septembre 2015, Milka c. Pologne, $\mathrm{n}^{\circ}$ 14322/12, $\$ 48$.

45. Cour EDH, 30 juin 2015, Khoroshenko c. Russie, $n^{\circ}$ 41418/o4.

46. Cour EDH, 18 mars 2014, Öcalan c. Turquie, $\$ 119$

47. Ibid., $\$ 120$ sq.

48. Voir également, inter alia, Cour EDH, 18 juin 2015, Ushakov et Ushakova c. Ukraine, nº 10705/12; 17 novembre 2015, Seyfettin Günes c. Turquie $n^{\circ} 22182 / 10$ 
retiennent ainsi une double violation des articles 3 et 6 de la Convention en raison d'une procédure particulièrement inacceptable. Le requérant ayant été condamné à une peine d'emprisonnement de quatorze ans pour le meurtre d'un adolescent, la Cour suprême avait confirmé le jugement initial en jugeant notamment que sa culpabilité avait été suffisamment établie par les déclarations à charge qu'il avait faites au cours de l'enquête préliminaire. Or ces pseudo-aveux (alors qu'il n'était entendu que comme témoin dans cette affaire pénale) avaient été obtenus sous la contrainte, tant physique que psychologique, et sans la présence d'un avocat. La procédure à l'encontre de M. Petrenko avait donc été particulièrement inéquitable ${ }^{49}$. Les faux aveux ne sont pas plus admissibles que les déclarations de culpabilité avant un jugement, comme le relève l'arrêt Neagoe c. Roumanie du 21 juillet 2015 (n² 23319/o8) dans lequel la Cour rappelle qu'une déclaration du porteparole d'une cour d'appel avant le délibéré de cette cour, incitant le public à croire à la culpabilité du requérant, constitue une violation du droit à la présomption d'innocence, qui doit être respecté avant le prononcé de toute décision de justice.

La Cour de Strasbourg exige également que le déroulement de la détention soit placé sous l'œil vigilant du juge interne.

Les détenus ont évidemment le droit de contester le fondement même de la détention, dans le cadre d'un recours effectif ${ }^{50}$ qui respecte les exigences générales du droit au procès équitable, ce qui n'est évidemment pas le cas pour des audiences portant sur des recours dirigés contre la prolongation d'une détention provisoire tenues en l'absence du requérant, ou en l'absence de son avocat ${ }^{51}$. Ce contrôle du juge permet notamment de faire vérifier qu'une détention provisoire est fondée sur des motifs pertinents et suffisants ${ }^{52}$. Certaines situations exigent même que les juridictions internes agissent avec une célérité maximale, comme dans le cas d'une demande de mise en liberté émanant d'un détenu souffrant de pathologies graves ${ }^{53}$.

Les personnes privées de liberté doivent également posséder dans l'ordre interne des voies de recours adéquates leur permettant de se plaindre de leurs conditions de détention ${ }^{54}$. À ce titre, la Cour européenne s'est félicitée dans son arrêt Yengo c. France du 21 mai 2015 de la possibilité maintenant ouverte aux détenus d'utiliser la voie du référé-liberté devant le juge administratif, qui peut désormais

[...] prononcer des injonctions sur le fondement des articles 2 et 3 de la Convention, en vue de faire cesser rapidement des conditions de détention attentatoires à la dignité $[\ldots]^{55}$.

Mais ce satisfecit n'est pas complet, car les juges strasbourgeois déplorent également un contentieux judiciaire qui reste trop fermé, avec une Cour de cassation qui conditionne toute possibilité d'une mise en liberté dans des situations de détention indignes à l'existence «d'éléments propres à la personne concernée, suffisamment graves pour mettre en danger sa santé physique ou mentale $»^{56}$. De telles exigences confèrent à cette voie de recours un caractère accessible mais non effectif en pratique, car ne respectant pas les nécessaires exigences de célérité ${ }^{57}$. Le contrôle de la qualité de la détention est évidemment encore plus indispensable concernant les détenus les plus vulnérables (tels que des détenus atteints de troubles psychiatriques), comme le démontrent les arrêts du 3 février 2015, Smits et autres. c. Belgique ( $\mathrm{n}^{\circ}$ 49484/11, 53703/11, 4710/12, 15969/12, 49863/12 et 70761/12), dans lesquels la Cour condamne la Belgique pour n'avoir pas mis à disposition des requérants une voie de recours effective pour faire valoir le caractère inapproprié de leur détention ${ }^{58}$. Consciente de la faible mise en œuvre de ces arrêts par les États concernés, la Cour de Strasbourg a eu récemment recours à sa technique de l'arrêt pilote, dans ses arrêts Neshkov et autres c. Bulgarie du 27 janvier 2015 ( $n^{\circ} 36925 / 10,21487 / 12,72893 / 12,73196 / 12$, $77718 / 12$ et 9717/13). Ayant déjà tranché plus de vingt affaires comparables et étant saisie d'une quarantaine d'autres concernant les conditions de détention en Bulgarie, elle en a conclu que la situation revêtait le caractère d'un problème structurel, nécessitant dans les dix-huit mois une réforme du droit et de la pratique bulgares, afin de mettre en place des recours préventifs et compensatoires effectifs.

De telles voies de recours effectives doivent également être ouvertes aux fins de faire reconnaître et condamner des

49. Voir également les arrêts du 5 février 2015, Razzakov c. Russie, $\mathrm{n}^{\circ} 57519 / 09$, concernant des actes de torture en garde à vue à fin de faire avouer un meurtre, et du 18 juin 2015, Ushakov et Ushakova c. Ukraine. Dans son arrêt du 16 juin 2015, Schmid-Laffer c. Suisse, nº 41269/o8, la Cour rappelle toutefois que toute atteinte aux droits d'un accusé n'entraîne pas l'invalidité de la procédure. Ainsi, le fait de ne pas informer un accusé de ses droits de ne pas s'incriminer et de garder le silence ne viole pas l'article $6, \S 1$, de la Convention si le reste du procès dans son ensemble n'a pas été inéquitable.

50. Cour EDH, 16 juin 2015, Levent Bektaş c. Turquie, $\mathrm{n}^{\circ} 70026 / 10$.

51. Cour EDH, 3 avril 2014, Artemov c. Russie, $\mathrm{n}^{\circ}$ 14945/03.

52. Cour EDH, 12 juin 2014, Chuprikov c. Russie, n $17504 / 07$.

53. Cour EDH, 5 juin 2014, Christodoulou et autres c. Grèce, no 80452/12.

54. Cour EDH, 17 avril 2014, Kavouris et autres c. Grèce, $\mathrm{n}^{\circ} 73237 / 12$.

55. Cour EDH, 21 mai 2015, Yengo c. France, $\$$ 68. Voir CE, réf., 22 décembre 2012, Section française de l'Observatoire international des prisons, $\mathrm{n}^{\circ} 364584,364620,364621$ et 364647 .

56. Cass. crim., 29 février $2012, \mathrm{n}^{\circ} 11-88.441$

57. Sur le défaut de contestation des conditions de détention, voir Cour EDH, 17 avril 2014, Lici c. Grèce, $\mathrm{n}^{\circ}$ 69881/12.

58. Voir également Cour EDH, 16 juillet 2015, Kuttner c. Autriche, $n^{\circ}$ 7997/o8, concernant le défaut de célérité des juridictions internes qui ont dû examiner la requête d'un détenu condamné qui souhaitait être libéré d'une institution psychiatrique et transféré dans une prison ordinaire; 3 juillet 2015, Bataliny c. Russie, n 1006o/07. 
mauvais traitements perpétrés par des codétenus ${ }^{59}$, pour contester une mesure de transfert dans une prison où le requérant a été soumis au régime de détention le plus sévère, alors qu'il avait droit à un régime moins strict ${ }^{60}$, ou encore pour faire vérifier de manière régulière le bien-fondé d'une peine perpétuelle. Dans les arrêts Harakchiev et Tolumov c. Bulgarie du 8 juillet 2014 ( $\mathrm{n}^{\circ}$ 15018/11 et 61199/12), la Cour réaffirme en effet qu'une peine de réclusion à perpétuité peut être conforme à l'article 3 de la Convention européenne des droits de l'homme s'il existe, d'une part, une chance de libération et, d'autre part, une possibilité de contrôle à partir du moment où elle s'applique ${ }^{61}$. De plus, ces condamnés à vie ont le droit de savoir ce qu'ils doivent faire pour que leur libération puisse être envisagée et dans quelles conditions. La Cour met ici à nouveau en œuvre la technique de l'arrêt pilote, en indiquant à la Bulgarie qu'elle doit réformer, de préférence par la voie législative, le cadre juridique régissant le régime d'incarcération applicable aux personnes condamnées à une peine perpétuelle avec ou sans libération conditionnelle (et en particulier supprimer l'automaticité de l'infliction d'un régime de détention extrêmement rigoureux et de l'isolement à tous les détenus condamnés à la prison à vie).

Les États ne doivent enfin pas oublier que les détenus sont des requérants potentiels devant la Cour européenne des droits de l'homme, comme le rappelle l'arrêt Vorobyev c. Ukraine du 16 octobre 2014 ( $\left.\mathrm{n}^{\circ} 28242 / 10\right)$, dans lequel l'État est condamné pour avoir refusé de transmettre à l'intéressé les pièces du dossier pénal qu'il avait demandées dans le cadre de sa requête devant la Cour de Strasbourg. Les membres du personnel d'un établissement pénitentiaire ne peuvent pas davantage ouvrir la correspondance d'un détenu avec la Cour européenne des droits de l'homme et conserver les pièces qui y étaient jointes, a fortiori si cette censure s'accompagne de menaces et du refus de faire parvenir le courrier à la Cour de Strasbourg ${ }^{62}$.

L'existence de voies de recours ne suffit pas pour que la justice pénètre les portes des prisons. Les préjudices subis par les détenus doivent également être reconnus comme de vrais préjudices, et non pas comme de simples dommages collatéraux d'une détention. Ainsi en est-il notamment de la possibilité d'engager un recours et d'obtenir réparation à la suite d'une contamination par le VIH dans le cadre de soins prodigués dans un établissement pénitentiaire ${ }^{63}$. Les arrêts Milić et Nikezić c. Monténégro du 28 avril 2015, relatifs à des mauvais traitements commis par des gardiens sur les requérants lors de la fouille d'une cellule, rappellent par ailleurs que les réparations accordées doivent être adéquates, ce qui n'était pas le cas en l'espèce, puisque la Cour suprême (dans le cadre de la procédure d'indemnisation à laquelle ont tout de même eu accès les deux requérants) s'était limitée à reconnaître qu'ils avaient subi des «actes dégradants pour la dignité humaine ${ }^{64}$, ce qui n'avait pas permis de leur accorder une réparation d'un montant suffisant. Dans tous ces cas, ces recours en indemnisation doivent être suffisamment accessibles et ne pas être soumis à des exigences matériellement irréalisables par le requérant, comme le paiement des frais de justice, la nécessité d'étayer les demandes et le délai fixé pour remplir ces conditions (20 février 2014, Shishkovc. Russie, $\left.n^{\circ} 26746 / 05\right)$

Pour conclure cette chronique, il ne faut pas oublier que l'ensemble de ce système est fragile, car il repose sur un seul élément: la mise en œuvre des arrêts par les quarante-sept États membres du Conseil de l'Europe. Les affaires Firth et autres c. Royaume-Uni ( $\mathrm{n}^{\circ}$ 47784/o9 et 9 autres) et $\mathrm{McHugh}$ et autres c. Royaume-Uni du 10 février 2015 ( ${ }^{\circ}$ 51987/o8 et 1014 autres) démontrent bien les limites du système: alors que depuis l'arrêt Hirst c. Royaume-Uni ( $\left.n^{\circ} 2\right)$ du 6 octobre $2005\left(n^{\circ} 74025 / 01\right)$ la Cour tente d'imposer à cet État la suppression de la législation qui prévoit un retrait automatique du droit de vote pour toute personne incarcérée, elle constate dans ces deux décisions que, dix ans après, les autorités britanniques n'ont toujours pas souhaité mettre en œuvre les exigences de la Cour. Les arrêts pilotes Greens et M. T. c. Royaume-Uni du 23 novembre 2010 (n 60041/o8 et 60054/08) n'ont donc servi à rien, si ce n'est à radicaliser une partie de la classe politique britannique à l'encontre du système conventionnel...

59. Cour EDH, 3 mars 2015, M. C. c. Pologne, no 23692/o9.

60. Cour EDH, 16 décembre 2014, Dmitrijevs c. Lettonie, $\mathrm{n}^{\circ}$ 49037/o9.

61. Voir également Cour EDH, 15 septembre 2015, Kaytan $c$. Turquie, $\mathrm{n}^{\circ}$ 27422/o5

62. Cour EDH, 19 juin 2014, Shekhov c. Russie, n 12440/o4. Voir également 12 mars 2015, Kopanitsyn c. Russie, nº 43231/o4, pour une requête destinée à la Cour européenne des droits de l'homme et expédiée "par erreur» au cabinet du président de la Fédération de Russie et ensuite examinée et jugée infondée par le parquet de Moscou...; voir également 15 septembre 2015, Shishanov c. Moldavie, n 11353/o6.

63. Cour EDH, 9 janvier 2014, Gorelov c. Russie, $\mathrm{n}^{\circ}$ 49072/11.

64. Cour EDH, 28 avril 2015, Milić et Nikezić c. Monténégro, $\$ 42$. 